\title{
HOPF-LIKE BIFURCATIONS IN PLANAR PIECEWISE LINEAR SYSTEMS
}

\author{
Emilio Freire, Enrique Ponce and Francisco Torres
}

\begin{tabular}{ll} 
Abstract & \\
\cline { 2 - 2 } & Continuous planar piecewise linear systems with two linear zones \\
are considered. Due to their low differentiability specific tech- \\
niques of analysis must be developed. Several bifurcations giving \\
rise to limit cycles are pointed out.
\end{tabular}

\section{Introduction and preliminary results}

We are interested in nonlinear planar oscillators of the form

$$
\begin{aligned}
& \dot{x}=f(x)-y, \\
& \dot{y}=x-b y
\end{aligned}
$$

where $b \in \mathbf{R}$ and the function $f$ gives account of the nonlinearity. System (1) exhibits a structure very common in the oscillator's field: the $x$-dynamics could be viewed, under an adequate selection of $f(x)$, as a bistable system with a negative feedback represented by the $y$-state, which in turn has a linear first order dynamics. Examples of systems modelled by equation (1) appear in different domains: van der PolDuffing oscillators in mechanics and electronics, Fitzhugh [1]-Nagumo [2] equations of nerve conduction, Boissonade-De Kepper [3] equations of chemical oscillating reactions, among others.

In the study of such oscillators, one finds some situations where the nonlinearities involved are correctly modelled by piecewise linear functions with three linear pieces $[\mathbf{4}],[\mathbf{5}]$. To understand the corresponding dynamics, it is important to first consider the two linear pieces case, because some phenomena encountered in this configuration are inheritated by the more general case of three pieces [6].

Thus, we are naturally led to consider continuous planar piecewise linear systems with two linear zones, hereafter denoted 2CPL systems. 
In this situation, by means of rigid motions, we can consider without loss of generality that the boundary between the two zones coincides with the vertical axis. So we write

$$
\dot{\mathbf{x}}=\mathbf{F}(\mathbf{x})= \begin{cases}\mathbf{A}_{L} \mathbf{x}+\mathbf{c}_{L}, & \text { if } x_{1} \leq 0, \\ \mathbf{A}_{R} \mathbf{x}+\mathbf{c}_{R}, & \text { if } x_{1}>0,\end{cases}
$$

where $\mathbf{x}=\left(x_{1}, x_{2}\right) \in \mathbf{R}^{2}, \mathbf{A}_{L}, \mathbf{A}_{R}$ are $2 \times 2$ matrices and $\mathbf{c}_{L}, \mathbf{c}_{R} \in$ $\mathbf{R}^{2}$. Here the dot denotes derivatives with respect to $\tau$. The continuity requirement implies that $\mathbf{c}_{L}=\mathbf{c}_{R}=\mathbf{c}$ and the matrices $\mathbf{A}_{L}, \mathbf{A}_{R}$ must have the same second column, i.e. our systems become

$$
\begin{aligned}
\dot{\mathbf{x}}=\mathbf{F}(\mathbf{x}) & = \begin{cases}\mathbf{A}_{L} \mathbf{x}+\mathbf{c}, & \text { if } x_{1} \leq 0, \\
\mathbf{A}_{R} \mathbf{x}+\mathbf{c}, & \text { if } x_{1}>0,\end{cases} \\
\mathbf{A}_{L} & =\left(\begin{array}{ll}
l_{11} & a_{12} \\
l_{21} & a_{22}
\end{array}\right), \\
\mathbf{A}_{R} & =\left(\begin{array}{ll}
r_{11} & a_{12} \\
r_{21} & a_{22}
\end{array}\right) .
\end{aligned}
$$

By using scalar equations, we have equivalently

$$
\begin{aligned}
& \dot{x}_{1}=\varphi_{1}\left(x_{1}\right)+a_{12} x_{2}+c_{1}, \\
& \dot{x}_{2}=\varphi_{2}\left(x_{1}\right)+a_{22} x_{2}+c_{2},
\end{aligned}
$$

with

$$
\varphi_{i}\left(x_{1}\right)=\left\{\begin{array}{ll}
l_{i 1} x_{1}, & \text { if } x_{1} \leq 0, \\
r_{i 1} x_{1}, & \text { if } x_{1}>0,
\end{array} \text { and } i=1,2 .\right.
$$

For system (2), we are mainly interested in the bifurcations giving rise to limit cycles. If we define the parameters $t_{L}=\operatorname{trace}\left(\mathbf{A}_{L}\right)$ and $t_{R}=\operatorname{trace}\left(\mathbf{A}_{R}\right)$ and denote by $\operatorname{int}($.$) the interior region of a closed$ Jordan curve in the plane, then a first result concerning periodic orbits is the following. It can be easily deduced from Green's formula.

Proposition 1. If $\Gamma$ is a closed orbit of system (2) then

$$
\iint_{\operatorname{int}_{L}(\Gamma)} t_{L} d x_{1} d x_{2}+\iint_{\operatorname{int}_{R}(\Gamma)} t_{R} d x_{1} d x_{2}=t_{L} \cdot S_{L}+t_{R} \cdot S_{R}=0,
$$

where $\operatorname{int}_{L}(\Gamma)=\operatorname{int}(\Gamma) \cap\left\{x_{1}<0\right\}, \operatorname{int}_{R}(\Gamma)=\operatorname{int}(\Gamma) \cap\left\{x_{1}>0\right\}$,

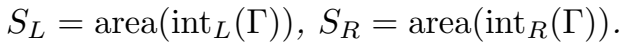

From Proposition 1, the following result about necessary conditions for the existence of isolated closed orbits, that is limit cycles, can be stated. 
Proposition 2. If $\Gamma$ is a limit cycle of system (2) then $\Gamma$ lives in the two zones, and the conditions $t_{L} \cdot t_{R}<0$ and $a_{12} \neq 0$ hold.

Proof: Within each zone, system (2) is linear and linear systems cannot have limit cycles, therefore $\Gamma$ lives in both zones.

If $t_{L} \cdot t_{R}>0$, from Proposition 1, system (2) cannot have periodic orbits (this is an analogous of Bendixson's criterion). As $\Gamma$ lives in the two zones and so $S_{L} \cdot S_{R}>0$, if one of the traces is zero, then the other trace value is also zero. This leads to a conservative system where no limit cycles are possible, getting a contradiction.

As is well known [7], every periodic orbit in a continuous planar vector field must contain at least one equilibrium in its interior. Then, if $a_{12}=0$, system (2) is uncoupled and the equilibrium in the interior of $\Gamma$ should have an associated invariant vertical straight line, which precludes the existence of periodic orbits.

We now give a canonical form to which all 2CPL systems capable of presenting limit cycles can be reduced.

Proposition 3. If a $2 C P L$ system has a limit cycle, then the system can be transformed into the following canonical form

$$
\begin{aligned}
& \dot{x}=f(x)-y, \\
& \dot{y}=x-b y-a,
\end{aligned} \text { where } f(x)= \begin{cases}\mu_{L} x, & \text { if } x \leq 0 \\
\mu_{R} x, & \text { if } x>0\end{cases}
$$

and the dot now denotes derivatives respect to a new variable s.

Proof: From Proposition 2, we can assume that $t_{L} \neq t_{R}$ and $a_{12} \neq 0$. Now, if we do in (3) a change of variables of the form

$$
\begin{aligned}
& u=x_{1}, \\
& v=\alpha x_{1}-a_{12} x_{2}-c_{1},
\end{aligned}
$$

then the vertical boundary is preserved. In the new variables, we have

$$
\begin{aligned}
& \dot{u}=\varphi_{1}(u)+\alpha u-v \\
& \dot{v}=\alpha \varphi_{1}(u)-a_{12} \varphi_{2}(u)+\left(\alpha-a_{22}\right)(\alpha u-v)+a_{22} c_{1}-a_{12} c_{2} .
\end{aligned}
$$

Considering the second equation piecewise linear term,

$$
\alpha \varphi_{1}(u)-a_{12} \varphi_{2}(u)= \begin{cases}\left(\alpha l_{11}-a_{12} l_{21}\right) u, & \text { if } u \leq 0 \\ \left(\alpha r_{11}-a_{12} r_{21}\right) u, & \text { if } u>0\end{cases}
$$


if we select

$$
\alpha=\frac{l_{21}-r_{21}}{l_{11}-r_{11}} a_{12}=\frac{l_{21}-r_{21}}{t_{L}-t_{R}} a_{12},
$$

the second equation in (5) is linear. We get a system of the form

$$
\begin{aligned}
& \dot{u}=g(u)-v, \\
& \dot{v}=\rho u+\sigma v+\xi,
\end{aligned} \text { where } g(u)= \begin{cases}m \cdot u, & \text { if } u \leq 0 \\
n \cdot u, & \text { if } u>0\end{cases}
$$

and $m-n=t_{R}-t_{L}$. If $\rho \leq 0$, a simple computation shows that the possible equilibria have real eigenvalues and, consequently, the system has invariant straight lines and no periodic orbits. Thus, we can assume $\rho>0$ in (6) and from the change,

$$
x=\sqrt{\rho} u, y=v, \tau=\frac{s}{\sqrt{\rho}}, a=-\frac{\xi}{\sqrt{\rho}}, b=-\frac{\sigma}{\sqrt{\rho}}, \mu_{L}=\frac{m}{\sqrt{\rho}}, \mu_{R}=\frac{n}{\sqrt{\rho}},
$$

the conclusion follows.

We remark that canonical form (4) is, modulo a translation of the variables, a system of form (1) which constitutes the main motivation of this work. Thus, system (1) includes all the systems with two-zones piecewise linear nonlinearities which can exhibit limit cycle behaviour.

In this paper, we aim to study for the canonical form (4) its Hopflike bifurcations, that is bifurcations that involve an equilibrium and a limit cycle. To do so, we introduce the parameters for the trace and the determinant in each zone,

$$
\begin{aligned}
t_{\Lambda} & =\mu_{\Lambda}-b, \\
d_{\Lambda} & =1-b \mu_{\Lambda},
\end{aligned}
$$

where $\Lambda=L, R$, standing for left or right, respectively.

We will assume $d_{L}, d_{R}>0$, which implies that system (4) has only one equilibrium point. Also, we will restrict ourselves to the case where

$$
D_{\Lambda}=d_{\Lambda}-\frac{t_{\Lambda}^{2}}{4}>0, \text { for } \Lambda=L, R
$$

that is, we have linear focus dynamics in both zones. This case will be named the focus-focus case. Other cases will appear elsewhere.

In the next section, we include the main results about Hopf-like bifurcations of codimension one in a certain three parameter space, showing four surfaces associated to three different kind of such bifurcations. These codimension one bifurcations surfaces are arranged around four 
codimension two curves emanating from one codimension three bifurcation point.

The study of periodic oscillations in piecewise linear planar system traces back to the Andronov's school. In fact, part of this work closely follows Andronov et al. [8], but we fill some gaps there, and we give, under the previous assumptions, the general bifurcation set for two-zones piecewise linear oscillators.

\section{Hopf-like bifurcations}

In this section, we give for the focus-focus case the main results of the paper about the bifurcations which are associated to changes of stability of an equilibrium point along with the appearance of a limit cycle. We call these phenomena Hopf-like bifurcations because they have the qualitative ingredients of differentiable Hopf bifurcations but, as it will be seen, there arise some dissimilarities.

We need to write the solutions for each zone, under our assumptions of having only one equilibrium point and linear focus dynamics in both zones. Namely,

$$
\left(\begin{array}{l}
x(s)-\frac{a}{d_{\Lambda}} \\
y(s)-\mu_{\Lambda} \frac{a}{d_{\Lambda}}
\end{array}\right)=e^{s \frac{t_{\Lambda}}{2}} C(s)\left(\begin{array}{l}
x(0)-\frac{a}{d_{\Lambda}} \\
y(0)-\mu_{\Lambda} \frac{a}{d_{\Lambda}}
\end{array}\right),
$$

where the matrix $C(s)$ is

$$
C(s)=\left(\begin{array}{cc}
\cos \left(\omega_{\Lambda} s\right)+\frac{\left(b+\mu_{\Lambda}\right) \sin \left(\omega_{\Lambda} s\right)}{2 \omega_{\Lambda}} & -\frac{\sin \left(\omega_{\Lambda} s\right)}{\omega_{\Lambda}} \\
\frac{\sin \left(\omega_{\Lambda} s\right)}{\omega_{\Lambda}} & \cos \left(\omega_{\Lambda} s\right)-\frac{\left(b+\mu_{\Lambda}\right) \sin \left(\omega_{\Lambda} s\right)}{2 \omega_{\Lambda}}
\end{array}\right)
$$

and $\omega_{\Lambda}=\sqrt{D_{\Lambda}}$, for $\Lambda=L, R$, standing for left or right zone, respectively. We also introduce two parameters which are crucial for the following analysis, namely the weighted traces

$$
\gamma_{L}=\frac{\mu_{L}-b}{2 \omega_{L}}=\frac{t_{L}}{2 \omega_{L}},
$$

$$
\gamma_{R}=\frac{\mu_{R}-b}{2 \omega_{R}}=\frac{t_{R}}{2 \omega_{R}} .
$$

If $a=0$, then the equilibrium is at the origin and so in the boundary between the two linear zones. Its topological type is determined in the next proposition. 
Proposition 4. Considering system (4) under the assumptions $D_{L}, D_{R}>0$, and $a=0$, the following statements hold.

(a) If $\gamma_{R}+\gamma_{L}>0$, the origin is a globally repulsive nonlinear focus.

(b) If $\gamma_{R}+\gamma_{L}=0$, the origin is a global nonlinear center.

(c) If $\gamma_{R}+\gamma_{L}<0$, the origin is a globally atractive nonlinear focus.

Proof: It suffices to compute a Poincaré return map $P$ on the halfstraight line $S_{+}=\{x=0, y \geq 0\}$. The map $P$ is built by a composition of the mapping $P_{L}$, which applies points of $S_{+}$into points of $S_{-}=$ $\{x=0, y \leq 0\}$ using the orbits in the region $x<0$, and the mapping $P_{R}: \mathbf{R}^{+} \rightarrow \mathbf{R}^{+}$, which applies points of $S_{-}$into points of $S_{+}$using the orbits in the region $x>0$, see Figure 1a.

Taking in (7) and (8) the values $a=0, x(0)=0, y(0)=p$, and working in the left zone, for $s_{L}=\pi / \omega_{L}$ we obtain $q=-y\left(s_{L}\right)=P_{L}(p)=$ $p \exp \left(\gamma_{L} \pi\right)$.

In an analogous way, putting $a=0, x(0)=0, y(0)=-q$ and working in the right zone, it turns out for $s_{R}=\pi / \omega_{R}$ that $r=P_{R}(q)=$ $q \exp \left(\gamma_{R} \pi\right)$. Hence,

$$
P(p)=e^{\left(\gamma_{L}+\gamma_{R}\right) \pi} p,
$$

and statements (a)-(c) follow easily.

When $a \neq 0$ in (4) the equilibrium point is located in the interior of one zone. The flow in this zone is linear, but the global flow is nonlinear and, as we will see now, a limit cycle can appear when $t_{L} \cdot t_{R}<0$. The next result closely follows the ideas used in Andronov et al. [8], which also appeared in Ye $[\mathbf{9}]$. However, in both references there are some little gaps and so we include its complete proof.

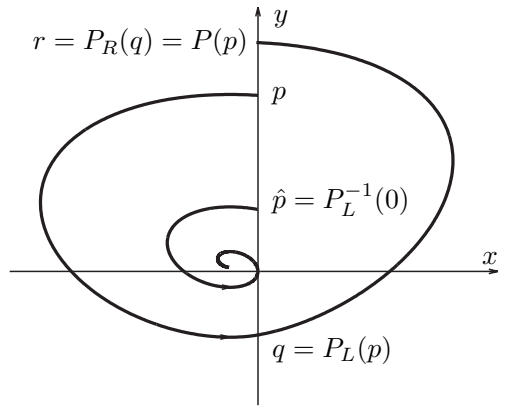

(a)

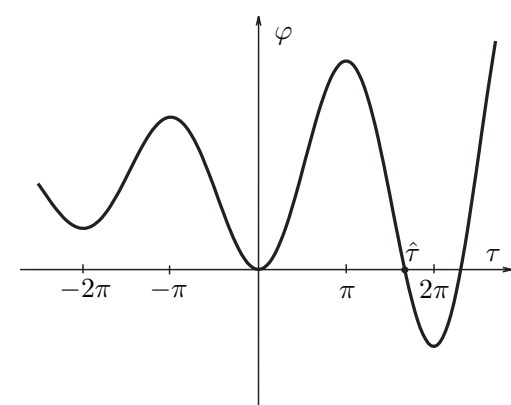

(b)

Figure 1. (a) Poincaré maps used through the paper. (b) Graph of function $\varphi$ for $\gamma>0$. 
Theorem 5. Consider system (4) with $D_{L}, D_{R}>0, a \neq 0$, and $t_{L} \cdot t_{R}<0$. Then there is only one equilibrium point and the following statements hold.

(a) If $\gamma_{L}+\gamma_{R}>0$ and $\operatorname{sign}(a)=\operatorname{sign}\left(\gamma_{L}\right)$, then the equilibrium is asymptotically stable, and it is surrounded by a unique unstable limit cycle.

(b) If $\gamma_{L}+\gamma_{R} \leq 0$ and $\operatorname{sign}(a)=\operatorname{sign}\left(\gamma_{L}\right)$, then the equilibrium is globally asymptotically stable and no limit cycles exist.

(c) If $\gamma_{L}+\gamma_{R} \geq 0$ and $\operatorname{sign}(a)=\operatorname{sign}\left(\gamma_{R}\right)$, then the equilibrium is unstable and no limit cycles exist.

(d) If $\gamma_{L}+\gamma_{R}<0$ and $\operatorname{sign}(a)=\operatorname{sign}\left(\gamma_{R}\right)$, then the equilibrium is unstable and it is surrounded by a unique stable limit cycle.

Moreover, when one limit cycle exists, its amplitude depends linearly on the parameter "a" and its period does not depend at all on that parameter.

Proof: Note that the equilibrium is in the left zone for $a<0$ and when $a>0$ it is in the right zone. We shall begin the proof by choosing $a<0$ and considering only assertions (a) and (b). Thus we have that $\gamma_{L}<0$. So $t_{L}<0$, and the equilibrium is an asymptotically stable focus located in the left zone.

To study the existence of limit cycles we now compute a Poincaré map $P$, which will be obtained in parametric form by means of certain phase angles. To this end let us introduce the auxiliary function,

$$
\varphi_{\gamma}(\tau)=1-e^{\gamma \tau}(\cos \tau-\gamma \cdot \sin \tau)
$$

with the symmetry properties

$$
\begin{aligned}
\varphi_{-\gamma}(-\tau) & =\varphi_{\gamma}(\tau), \quad \forall \gamma, \tau \in \mathbf{R} \\
\varphi_{-\gamma}(\tau) & =\varphi_{\gamma}(-\tau), \quad
\end{aligned}
$$

and the graph of Figure 1b. Note that for $\gamma>0$, the function $\varphi_{\gamma}$ has a first positive zero for a certain value $\hat{\tau}=\hat{\tau}(\gamma) \in(\pi, 2 \pi)$, and relative maxima at $\tau=-\pi, \tau=\pi$.

Taking now $(x(0), y(0))=(0,-q)$, with $q>0$ (that is, a point of $S_{-}$, as defined before) as initial point of an orbit, it is concluded that the trajectory evolves in the rigth zone until it eventually reaches the left zone. Note that the flow on $S_{-}$(respectively, $S_{+}$) is pointing to the right (respectively, to the left) and so the trajectory leaves the right zone in a point $(0, r)$ of $S_{+}$, after a time $s_{R}$. From (7) and (8) for $s=s_{R}$ and 
solving for $q$ and $r$, we can write,

$$
\begin{aligned}
& q\left(\tau_{R}\right)=-\frac{a \omega_{R} e^{-\gamma_{R} \tau_{R}} \varphi_{\gamma_{R}}\left(\tau_{R}\right)}{d_{R} \cdot \sin \tau_{R}} \\
& r\left(\tau_{R}\right)=-\frac{a \omega_{R} e^{\gamma_{R} \tau_{R}} \varphi_{-\gamma_{R}}\left(\tau_{R}\right)}{d_{R} \cdot \sin \tau_{R}},
\end{aligned}
$$

where $\tau_{R}=\omega_{R} s_{R}$, and $\tau_{R} \in(0, \pi)$. When $\tau_{R}$ varies from 0 to $\pi$, the functions $q\left(\tau_{R}\right)$ and $r\left(\tau_{R}\right)$ increase monotonically from 0 to $\infty$.

From (10) we can implicitely define the half-return map on the rigth zone as the $C^{\infty}$ map $P_{R}: \mathbf{R}^{+} \rightarrow \mathbf{R}^{+}$, with $P_{R}(q)=r$, and $P_{R}(0)=0$, see Figure 1a.

Integrating backwards from the point $(0,-q)$ on $S_{-}$, we analogously obtain a first intersection point $(0, p) \in S_{+}$after a time $-s_{L}$. Again from (7) and (8) and $s=-s_{L}$, we can solve for $p$ and $q$ and write,

$$
\begin{aligned}
& p\left(\tau_{L}\right)=\frac{a \omega_{L} e^{-\gamma_{L} \tau_{L}} \varphi_{\gamma_{L}}\left(\tau_{L}\right)}{d_{L} \cdot \sin \tau_{L}} \\
& q\left(\tau_{L}\right)=\frac{a \omega_{L} e^{\gamma_{L} \tau_{L}} \varphi_{-\gamma_{L}}\left(\tau_{L}\right)}{d_{L} \cdot \sin \tau_{L}}
\end{aligned}
$$

where $\tau_{L}=\omega_{L} s_{L} \in(\pi, \hat{\tau}]$. Note that $\varphi_{-\gamma_{L}}(\hat{\tau})=0$, and so $q(\hat{\tau})=0$. We will denote $\hat{p}=p(\hat{\tau})$. When $\tau_{L}$ decreases from $\hat{\tau}$ to $\pi$, the function $p\left(\tau_{L}\right)$ increases monotonically from $\hat{p}$ to $\infty$ and the function $q\left(\tau_{L}\right)$ from 0 to $\infty$.

For representing the half-return map with time reversal on the left zone, we define the $C^{\infty}$ map $P_{L}^{-1}: \mathbf{R}^{+} \rightarrow \mathbf{R}^{+}$, where $P_{L}^{-1}(q)=p>\hat{p}$ and $P_{L}^{-1}(0)=\hat{p}$, see Figure 1a.

Elementary calculations show that the function $p=P_{L}^{-1}(q)$ has the following derivatives,

$$
\begin{aligned}
\frac{d p}{d q} & =\frac{\varphi_{-\gamma_{L}}\left(\tau_{L}\right)}{\varphi_{\gamma_{L}}\left(\tau_{L}\right)} \\
\frac{d^{2} p}{d q^{2}} & =-\frac{2 d_{L}\left[1+\left(\gamma_{L}\right)^{2}\right] \sin ^{3} \tau_{L}}{a \omega_{L}\left[\varphi_{\gamma_{L}}\left(\tau_{L}\right)\right]^{3}}\left[\sinh \left(\gamma_{L} \tau_{L}\right)-\gamma_{L} \sin \tau_{L}\right]
\end{aligned}
$$

Now using that $\tau_{L} \in(\pi, \hat{\tau}]$ and the properties of the function $\varphi_{\gamma}$, the function $p(q)$ is increasing with

$$
\lim _{q \rightarrow \infty} p^{\prime}(q)=e^{-\gamma_{L} \pi} \text { and } p^{\prime \prime}(q)>0, \text { for } q \geq 0 .
$$


So we have that $0 \leq p^{\prime}(q)<e^{-\gamma_{L} \pi}$ for $q \geq 0$ and consequently, the inverse map $P_{L}$ is well defined and differentiable in the interval $(\hat{p}, \infty)$.

The expressions of the derivatives of the function $r=r(q)$ are identical to (12) if we change all subscripts from $R$ to $L$. So taking into account that $\tau_{R} \in(0, \pi)$, the function $r(q)$ turns out to be increasing with

$$
\lim _{q \rightarrow 0} r^{\prime}(q)=1, \lim _{q \rightarrow \infty} r^{\prime}(q)=e^{\gamma_{R} \pi} \text { and } r^{\prime \prime}(q)>0 \text {, for } q \geq 0 .
$$

We deduce that $1<r^{\prime}(q)<e^{\gamma_{R} \pi}$.

We can define the Poincaré map $r=P(p)=P_{R}\left(P_{L}(p)\right)$, where $P$ is defined from $[\hat{p}, \infty)$ to $[0, \infty)$, with $P(\hat{p})=0$. Accordingly we obtain by using (10)-(12),

(13) $\frac{d P}{d p}=\frac{\varphi_{\gamma_{L}}\left(\tau_{L}\right)}{\varphi_{-\gamma_{L}}\left(\tau_{L}\right)} \frac{\varphi_{\gamma_{R}}\left(\tau_{R}\right)}{\varphi_{-\gamma_{R}}\left(\tau_{R}\right)}=\frac{p}{P(p)} e^{2\left(\gamma_{L} \tau_{L}+\gamma_{R} \tau_{R}\right)}=\frac{p}{P(p)} e^{2 \nu(q)}$,

where $\nu(q)=\gamma_{L} \tau_{L}+\gamma_{R} \tau_{R}$, and $\tau_{L}, \tau_{R}$ are the phase angles of half-return maps through $q$.

Note that when $p \rightarrow \hat{p}^{+}$, then $\tau_{L} \rightarrow \hat{\tau}$ and $\tau_{R} \rightarrow 0$. If $p \rightarrow \infty$, then $\tau_{L} \rightarrow \pi$ and $\tau_{R} \rightarrow \pi$. Therefore from (13) we have

$$
\begin{aligned}
& \lim _{p \rightarrow \hat{p}^{+}} \frac{d P}{d p}=\infty, \\
& \lim _{p \rightarrow \infty} \frac{d P}{d p}=e^{\left(\gamma_{R}+\gamma_{L}\right) \pi} .
\end{aligned}
$$

We remark that if $\bar{p}$ is a fixed point of the map $P$, then (13) reduces to

$$
\left(\frac{d P}{d p}\right)_{p=\bar{p}}=e^{2 \nu(\bar{q})} \text {, where } \bar{q}=P_{L}(\bar{p}) .
$$

Moreover, as the functions $r(q)$ and $p(q)$ verify $r(0)=0<\hat{p}=p(0)$, by continuity we have $r(q)<p(q)$ for $q<\bar{q}$. It can be deduced that

$$
1 \leq \frac{r^{\prime}(\bar{q})}{p^{\prime}(\bar{q})}=\left(\frac{d P}{d p}\right)_{p=\bar{p}}=e^{2 v(\bar{q})}
$$

and so $\nu(\bar{q}) \geq 0$.

In our working case, the function $\nu(q)$ is strictly increasing because to a larger $q$ corresponds a larger $\tau_{R}$ and a smaller $\tau_{L}$. Therefore, this function can vanish only in one fixed point, at most. As a consequence, a continuum of fixed points cannot happen and the fixed points, if any, are isolated. 
Now, we will study the existence of fixed points of the map $P$ under the hypotheses of statement (a), by considering the function $h(p)=P(p)-p$ for $p \in[\hat{p}, \infty)$. We know that $h(\hat{p})=-\hat{p}<0$, and from (14) there exists $k>0$ such that for $p$ big enough we have $h^{\prime}(p)>k>0$, because we are assuming $\gamma_{R}+\gamma_{L}>0$. Hence, using adequately the mean value theorem, we deduce that $h(p)>0$ when $p$ is big enough. From the intermediate value theorem, a value $\bar{p}$ with $h(\bar{p})=P(\bar{p})-\bar{p}=0$, exists. Consequently, the map $P$ has at least one fixed point, i.e. system (4) has at least one limit cycle.

Suppose now that for the Poincaré map $P$ there exist two fixed points $\bar{p}_{1}$ and $\bar{p}_{2}$, corresponding to $\bar{q}_{1}<\bar{q}_{2}$ and no fixed points in the interval $\left(\bar{p}_{1}, \bar{p}_{2}\right)$. We must necessarily have

$$
\nu\left(\bar{q}_{1}\right)=0, \quad\left(\frac{d P}{d p}\right)_{\bar{p}_{1}}=1<\left(\frac{d P}{d p}\right)_{\bar{p}_{2}},
$$

because two consecutive fixed points with derivatives greater than 1 are not allowed. In this situation, for points $p$ to the left of $\bar{p}_{2}$ and sufficiently close to it, we have $p>P(p)$ and the same must be true for all $p \in$ $\left(\bar{p}_{1}, \bar{p}_{2}\right)$. The second factor of $(13)$ is also greater than 1 , so $P^{\prime}(p)>1$ for all $p \in\left(\bar{p}_{1}, \bar{p}_{2}\right)$, that leads to a contradiction with the mean value theorem. Therefore, the graph of the map $P$ has only one crossing with the bisector of the first quadrant that corresponds with an unstable limit cycle. Statement (a) is then true for $a<0$.

We study now the existence of fixed points of the map $P$ under the hypotheses of statement (b), keeping the assumption $a<0$. Thus, $\gamma_{L}<$ 0 and the equilibrium is also an asymptotically stable focus. Suppose that the corresponding Poincaré map $P$ has a fixed point $\bar{p}_{1}$. From (15) we must have $P^{\prime}\left(\bar{p}_{1}\right) \geq 1$, that is $v\left(\bar{q}_{1}\right) \geq 0$. But in this case, we have

$$
v\left(\bar{q}_{1}\right)=\gamma_{L} \tau_{L}+\gamma_{R} \tau_{R} \leq \gamma_{L}\left(\tau_{L}-\tau_{R}\right)<0,
$$

because $\gamma_{L}+\gamma_{R} \leq 0$ and $\tau_{L}>\pi>\tau_{R}$. We obtain a contradiction, and so the map $P$ has no fixed points and statement (b) is shown for $a<0$.

Statements (a) and (b) for $a>0$ and statements (c) and (d) for $a \neq 0$ can be reduced to some of the cases just studied, by using the symmetry properties of the system.

When the limit cycle exists, it can be determined by solving the equations

$$
\begin{aligned}
& q\left(\tau_{R}\right)=q\left(\tau_{L}\right), \\
& r\left(\tau_{R}\right)=p\left(\tau_{L}\right),
\end{aligned}
$$


where $\tau_{R} \in(0, \pi)$ and $\tau_{L} \in(\pi, \hat{\tau})$. Note that, from (10) and (11), the solution of above equations does not depend on the value of parameter $a$. In fact, keeping equal the other parameters, phase planes for two different values of $a$ with the same sign are homothetic. So, the final statement of the theorem is proved.

From Proposition 2 , when $t_{L} \cdot t_{R} \geq 0$, the existence of limit cycles is not possible, but we can have limited zones where a continuum of periodic orbits exists, as we will see in the next result. These configurations will be named zonal linear centers.

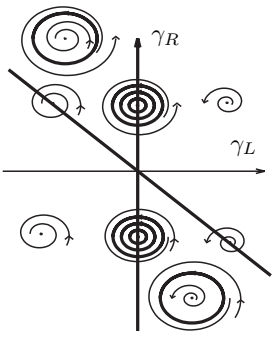

(a) $a<0$

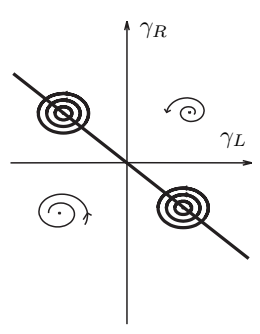

(b) $\quad a=0$

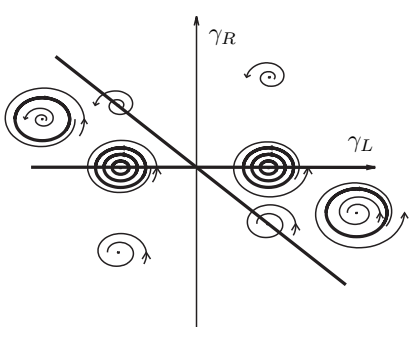

(c) $a>0$

Figure 2. Bifurcation sets in the plane $\left(\gamma_{L}, \gamma_{R}\right)$ for: (a) $a<0$, (b) $a=0$ (c) $a>0$. We sketch the different phase planes in each region.

Theorem 6. Consider system (4) with $D_{L}, D_{R}>0$ and $a \neq 0$. Then it has only one equilibrium point and the following statements hold.

(a) If $t_{L} \cdot t_{R}>0$ and the traces are negative (respectively, positive), then the equilibrium is a globally attractive (respectively, repulsive) focus.

(b) If $t_{L} \cdot t_{R}=0$ and both values are not zero, then the following cases arise.

(b1) When the equilibrium lies in a zone with negative (respectively, positive) trace, then it is a globally attractive (respectively, globally repulsive) focus.

(b2) When the equilibrium lies in the zone with trace zero and the other trace is negative (respectively, positive), system (4) has a globally attractive (respectively, repulsive) zonal linear center.

Proof: (a) The only equilibrium is locally a linear focus and in the two cases considered the traces in each zone have the same sign. From Proposition 2 no limit cycles can exist and the Poincaré map of the proof 
of Theorem 5 is well defined for every point $p>\hat{p}$ of $S_{+}$. Then the local stability character of the focus coincides with its global character.

(b1) The arguments of the proof of statement (a) are also valid for this case.

(b2) The equilibrium is a linear center and the closed orbits of the center configuration included in the zone with non-zero trace end with one closed orbit tangent to the $y$-axis at the origin. Using the Poincaré map of the proof of Theorem 5 , it is easily concluded that $P(0)=0$. Studying the stability of this fixed point, the conclusion follows.

Always assuming $D_{L}, D_{R}>0$, we can summarise the information supplied by Proposition 4 and Theorems 5 and 6 in Figures 2 and 3 .

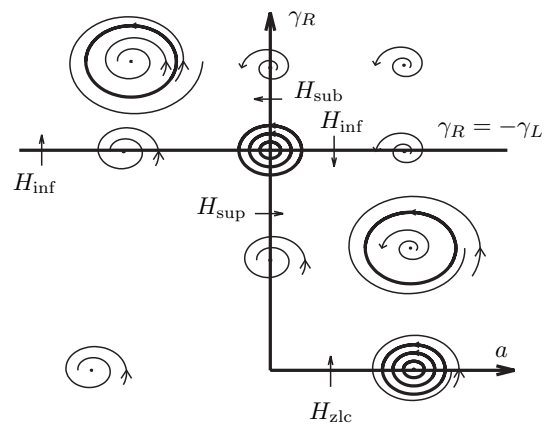

(a)

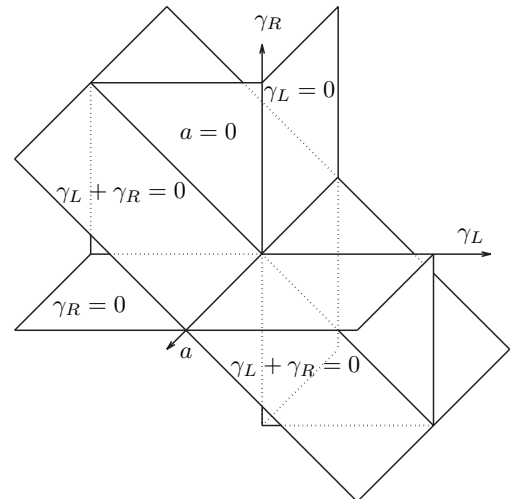

(b)

Figure 3. (a) Bifurcation set for $\gamma_{L}<0$ in the plane $\left(a, \gamma_{R}\right)$. (b) Bifurcation set in the space $\left(a, \gamma_{L}, \gamma_{R}\right)$. The origin constitutes a codimension three bifurcation point.

We want to emphasize that three different kinds of Hopf bifurcations arise from the developed analysis. Consider first, for instance, the situation when $\gamma_{L}<0, \gamma_{R}>0$, and $\gamma_{L}+\gamma_{R}<0$, see Figure 3a. If we select $a$ as the bifurcation parameter and move it from a negative value to a positive value, then a supercritical Hopf bifurcation takes place at $a=0$. Effectively, when $a<0$, from statement (b) of Theorem 5 the equilibrium is globally asymptotically stable. For $a>0$ it is unstable and a stable limit cycle exists, according to statement (d) of the same theorem. Analogously, when $\gamma_{L}<0, \gamma_{R}>0$, and $\gamma_{L}+\gamma_{R}>0$, a subcritical Hopf bifurcation happens at $a=0$ because then from statement (a) we have, for $a<0$, a stable equilibrium surrounded by a unstable limit cycle, and for $a>0$ the equilibrium is globally unstable, according to 
statement (c). These bifurcations are different from Hopf bifurcations for differentiable systems since here the amplitude of the limit cycle involved depends linearly on the bifurcation parameter and its period is constant.

A second kind of Hopf bifurcations occurs when a limit cycle is born from a zonal linear center. For instance, select fixed values $a>0$ and $\gamma_{L}<0$ and then move $\gamma_{R}$ from negative to positive values, see Figures $2 \mathrm{c}$ and 3a. From statement (b2) of Theorem 6 , at $\gamma_{R}=0$ we have an atractive zonal linear center that gives rise to a stable limit cycle for $0<\gamma_{R}<-\gamma_{L}$, according with statement (d) of Theorem 5. This bifurcation is characterized by a jump transition to an oscillatory state of significative amplitude.

The third kind of Hopf bifurcation arises for a fixed value of $a \neq 0$ at the parameter values such that $\gamma_{L}+\gamma_{R}=0$, by considering either the transition between the hypotheses of statements (a) and (b) of Theorem 5, or that corresponding to statements (c) and (d) of the same theorem. See Figures 2a, 2c and 3a. Then a limit cycle appears or dissapears without any change of stability of the equilibrium. This phenomenon corresponds to a Hopf bifurcation at infinity.

Four situations of codimension two appear. First, for $a \neq 0$ the points $\left(\gamma_{L}, \gamma_{R}\right)=(0,0)$. Second, every point $\left(a, \gamma_{R}\right)=\left(0,-\gamma_{L}\right)$ with $\gamma_{L} \neq 0$. A third situation of codimension two arises for the points $a=\gamma_{R}=0, \gamma_{L} \neq$ 0 . Finally, the points $a=\gamma_{L}=0$ with $\gamma_{R} \neq 0$ are also of codimension two.

We can describe all the possible situations in the three parameter space $\left(a, \gamma_{L}, \gamma_{R}\right)$, see Figure 3b. As indicated before, we find several surfaces of codimension one bifurcation points, namely, the quadrants of plane $a=0$ with $\gamma_{L} \gamma_{R}<0$, the plane $\gamma_{L}+\gamma_{R}=0$, and the half-planes $\gamma_{L}=0$ for $a<0$ and $\gamma_{R}=0$ for $a>0$. These surfaces intersect in the four lines of codimension two points which emanate from the point $\left(a, \gamma_{L}, \gamma_{R}\right)=(0,0,0)$, and so the origin of this parameter space represents a codimension three bifurcation point.

Acknowledgements. Second author is partially supported by DGICYT grant TAP94-0491.

\section{References}

1. R. FitzHugh, Impulses and physiological states in theoretical models of nerve membrane, Biophys. J. 1 (1961), 445-466. 
2. J. S. Nagumo, S. Arimoto and S. Yoshizawa, An active pulse transmission line simulating nerve axon, Proc. IRE. 50 (1962), 2061-2071.

3. J. Boissonade And P. De KePper, Transitions from bistability to limit cycle oscillations, J. Phys. Chem. 84 (1980), 501-506.

4. J. Llibre AND J. Sotomayor, Phase portrait of planar control systems, Nonlinear Anal. 27 (1996), 1177-1197.

5. G. A. Kriegsmann, The rapid bifurcation of the Wien bridge oscillator, IEEE Trans. Circuits Systems CAS 34 (1987), 1093-1096.

6. E. Freire, E. Ponce And F. Torres, Hopf bifurcations in piecewise linear planar dynamical systems, Proceedings NDES'96, Sevilla (1996), 129-134.

7. P. Hartman, "Ordinary differential equations," Birkäuser, Boston, 1982.

8. A. Andronov, A. Vitt And S. Khaikin, "Theory of oscillations," Pergamon Press, Oxford, 1966.

9. Yan-Qian Ye, "Theory of Limit Cycles," Trans. Math. Mon. 66, American Mathematical Society, Providence RI, 1986.

\author{
Departamento de Matemática Aplicada II \\ Escuela Superior de Ingenieros \\ Avda. Reina Mercedes \\ 41012 Sevilla \\ SPAIN \\ e-mail: emilio@matinc.us.es \\ e-mail: enrique@matinc.us.es \\ e-mail: torres@polimat01.eup.us.es
}

Primera versió rebuda el 30 de Novembre de 1996 , darrera versió rebuda el 19 de Febrer de 1997 